\title{
TERRESTRIAL RADAR AND LASER SCANNING FOR DEFORMATION MONITORING: FIRST STEPS TOWARDS ASSISTED RADAR SCANNING
}

\author{
D. Wujanz ${ }^{1}$, F. Neitzel ${ }^{1}$, H. P. Hebel ${ }^{2}$, J. Linke², W. Busch ${ }^{2}$ \\ ${ }^{1}$ Technische Universität Berlin \\ Institute of Geodesy and Geoinformation Science \\ Chair of Geodesy and Adjustment Theory \\ Strasse des 17. Juni, 10623 Berlin, Germany \\ daniel.wujanz | frank.neitzel@tu-berlin.de \\ ${ }^{2}$ Technische Universität Clausthal \\ Department of Geotechnical Engineering and Mine Surveying \\ Chair of Mine Surveying and Geoinformation \\ Erzstrasse 18, 38678 Clausthal-Zellerfeld, Germany \\ hans-peter.hebel | janette.linke | \\ wolfgang.busch@tu-clausthal.de
}

\section{Commission V}

KEY WORDS: Terrestrial Laser Scanning, Ground based RADAR, Deformation Analysis, Sensor Fusion

\begin{abstract}
:
Deformation analysis based on multi-temporal Terrestrial Laser Scanning (TLS) surveys has been applied for many years in commercial and academic problem domains. Downsides of this technique are the sequential data acquisition as well as its limited accuracy compared to terrestrial InSAR or InRAR based technologies that are currently gaining attention of the geodetic community. A drawback of these instruments is its relative operation method while beneficially measurements within the entire field of view can be carried out simultaneously at high frequencies. At first comparative studies between TLS and InRAR scanning are presented which have been carried out at a quarry. Over the course of roughly two hours six epochs have been measured where geometric changes of different degrees have been purposely made. In order to ensure comparability concerning the outcome, both instrument coordinate systems have been transformed into a common coordinate system by applying corner cube reflectors. At last an assisted ground based RADAR approach is presented where advantages of both applied techniques are incorporated.
\end{abstract}

\section{INTRODUCTION}

A central ambition of research in the field of geodetic data acquisition is to find ways in order to capture an object or area of interest in a spatially and temporally continuous fashion. The achievement of this goal would result in a quantum leap of understanding behaviour and characteristics of objects and the environment especially in the field of engineering surveying to which the problem of deformation analysis is associated. The last decade has been dominated, scientifically as well as commercially, by terrestrial laser scanning (TLS) which is capable of achieving spatial quasi-continuity, while the last mentioned aspect can be rated as a large advantage over conventional classical methodologies such as tacheometry. On the other hand it can be seen as a disadvantage since points captured on various objects can't unambiguously be assigned to each other - hence direct determination of deformation is not achievable. In order to nevertheless solve this problem several approaches have been developed respectively are still under development such as ELING (2009) or WUJANZ et al. (2013).

Recent attempts by applying ground-based RADAR (GBRADAR) in the field of deformation monitoring showed that this technology is very close in fulfilling the central ambition mentioned before. Another advantage is the fact that deformation is "directly" derivable from multi-temporal observations. The achievable precision of such systems can be assumed with less than $1 \mathrm{~mm}$. In contrast to TLS, where an object's surface is discretised by a point grid, RADAR interferometry captures its data by rasterising in laminar fashion. The size of a raster cell in line of sight (LOS) is dependent to bandwidth and angle of beam of the applied antenna if a real aperture RADAR (RAR) is used. As the bandwidth is constant a fixed resolution in depth direction is the consequence. The azimuthal resolution is dependent to the length of the applied antenna which leads to a certain angular resolution. Hence the resolution in this direction is dependent to distance. In case of synthetic aperture RADAR (SAR) a dependency to the length of an applied rail is present which is used to translate the instrument. The resulting size of a raster cell lies between $0.1 \mathrm{~m}$ and $50 \mathrm{~m}$.

However, a major problem of GB-RADAR is the comparison of subsequently captured epochs. Two reasons are responsible for the existence of this problem. The first one being the dependence of RADAR techniques to a certain standpoint while the second issue is related to solving ambiguities that are caused by deformations larger than half of the wavelength $(17.3 \mathrm{~mm} /$ 2) - the area of uniqueness. Solving for ambiguities is also referred to as phase unwrapping and is provoked by the relative measurement principle. Table 1 gathers a comparison of different aspects concerning both systems while the centre 
column comprises desired properties for a monitoring system. Simultaneously this column contains the motivation for this contribution which could be achieved by merging the stated advantages.

\begin{tabular}{|c|c|c|}
\hline & Advantage & Problems \\
\hline TLS & $\begin{array}{ll}\text { - } & \text { Absolute approach } \\
\text { - } & \text { Spatially laminar } \\
\text { - } & \text { Huasi-continuity } \\
\text { High range }\end{array}$ & $\begin{array}{ll}\text { - } & \text { Sequential data } \\
\text { acquisition } \\
\text { - } & \text { Poor / unknown } \\
\text { stochastic } \\
\text { models } \\
\text { - } \quad \text { Too simplified } \\
\text { post processing } \\
\text { software }\end{array}$ \\
\hline $\begin{array}{c}\text { GB- } \\
\text { RADAR }\end{array}$ & $\begin{array}{l}\text { Simultaneous and } \\
\text { high frequent data } \\
\text { acquisition } \\
\text { - } \quad \text { High range and } \\
\text { precision for } \\
\text { geometrical } \\
\text { changes (depth) } \\
\text { - Laminar continuity }\end{array}$ & $\begin{array}{ll}\text { - } & \text { Ambiguous for } \\
\text { larger } \\
\text { deformations } \\
\text { - } & \text { Complex post } \\
\text { processing } \\
\text { - } & \text { Linked to one } \\
\text { standpoint } \\
\text { - } & \text { Low spatial } \\
\text { resolution }\end{array}$ \\
\hline
\end{tabular}

A sound overview on GB-RADAR instruments in deformation monitoring is presented by RÖDELSPERGER (2011) exemplified on a landslide, a quarry, rock faces and a caldera flank. A brief comparative study of TLS and ground based RADAR has been presented by LUZI (2010). However, the focus of this contribution was set on the introduction of ground based RADAR interferometry in the context of geo-scientific monitoring. A comparative study between aerial RADAR based observations and aerial laser scanning (ALS) has been conducted by KAASALAINEN et al. (2010) though in the problem domain of forest defoliation. WERNER et al. (2008) introduce a portable ground based RADAR interferometer which has been applied in this contribution and is hence described in section 2.1. First experiences of slope monitoring by applying this instrument are presented by HeBEL et al. (2011). AlbA et al. (2009) apply a GB-RADAR system for monitoring of a dam and raise the idea to combine ground based RADAR systems with other ranging sensors such as TLS or robotic total stations in order to solve eventual issues caused by phase unwrapping. LiNDENBERGH \& PFEIFER (2005) apply TLS for deformation monitoring of lock doors. ABELLAN et al. (2009) detect millimetric changes by nearest neighbor averaging of TLS measurements. The contribution at hand features a comparative experimental study between GB-RADAR and TLS. Deformation monitoring is carried out by applying both sensors in a quarry where geometric changes have been deliberately conducted within the region of interest. The installed changes led to false results during the process of phase unwrapping which leads to the conclusion that RADAR instruments are not applicable for campaign-wise deformation monitoring. Hence the motivation arises to introduce additional information represented by TLS measurements of the area of interest in order to solve the existing problem. In addition a novel methodology, referred to as assisted GB-RADAR (aGBRADAR), is proposed to solve ambiguities which have been caused by deformation based on TLS measurements. Section 2 describes the applied sensors, the area of interest and features a comparative analysis of the outcome of both instruments. Section 3 introduces the new methodology while the last one concludes and gives an outlook on prospective work.

\section{COMPARATIVE ANALYSIS OF TLS AND GB-RADAR}

This section introduces the applied instruments as well as the area of interest. Subsequently deformation analysis is conducted solely based on each instrument's datasets. Data acquisition has been conducted from one standpoint for each instrument while the stability of each position has been frequently checked by remeasuring artificial targets that have been distributed over the area of interest. Furthermore a way of integrating both data sets into a common coordinate system is presented which describes the basis for a comparative view on the computed results.

\subsection{Applied instruments}

The applied terrestrial laser scanner is a Leica C10 instrument which utilises the time of flight principle and operates at $532 \mathrm{~nm}$ (LEICA 2013). Its maximum reach adds up to $300 \mathrm{~m}$ at $90 \%$ albedo while a rather plausible degree of reflection for the desired use of $18 \%$ allows distance measurements up to $134 \mathrm{~m}$. Stochastic information is oddly enough only provided for an object distance of up to $50 \mathrm{~m}$ which appears to be inappropriate since much higher distances can be measured. The spatial single point accuracy $(1 \sigma)$ is assumed to measure $6 \mathrm{~mm}$ while no specific influencing factors are mentioned. Since the provided information appears to be unsatisfactory an empirical stochastic model dependent on distance is employed which will be briefly described later. Furthermore a Swiss made GPRI-2 terrestrial RADAR scanner (TRS) by GAMMA remote sensing has been utilised. The instrument continuously emits frequencymodulated waves (FMCW) within the certified band width of 17.1-17.3 GHz approved by the European Union which leads to a wavelength of $17.3 \mathrm{~mm}$. The maximum operational range sums up to $6 \mathrm{~km}$ featuring an azimuthal resolution of $41.9 \mathrm{~m}$. For a levelled instrument, the angle of aperture amounts to $0.4^{\circ}$ in horizontal direction while the according vertical value is $60^{\circ}$. The range resolution in LOS adds up to $75 \mathrm{~cm}$ with a precision of less than $2 \mathrm{~mm}$ (WIESMANN et al. 2008). Figure 1 shows both instruments within the passive area of a quarry.

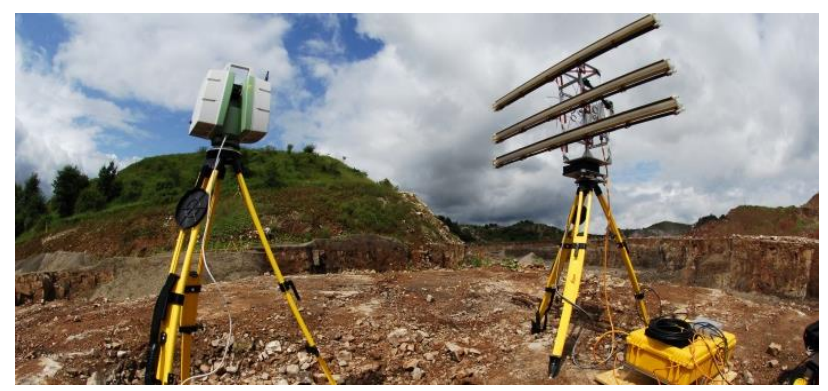

Figure 1: Leica C10 TLS (left) and GAMMA RADAR scanner (right)

\subsection{Description of the site of interest}

The site of interest is located at a quarry in the secondary mountains of the Harz region about $80 \mathrm{~km}$ south of Brunswick, Germany. Subject of the conducted survey was a slope where no drivage was carried out in the otherwise active pit. The measurements have been simultaneously accomplished by both instruments in six epochs where deformation has been deliberately introduced in between five measurements at different degrees by digging. Figure 2 illustrates the area of interest which is highlighted by a red polygon while tie points are marked by yellow shapes. 


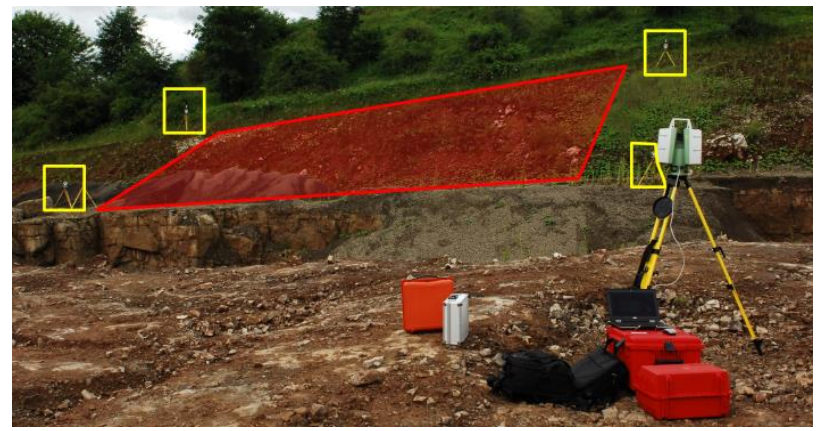

Figure 2: The area of interest is highlighted by a red polygon while four applied tie points are marked by yellow rectangles

While only one measurement was conducted with the TLS per epoch, continuous acquisition has been carried out by the TRS. The measurements were interrupted when geometric changes have been made in the area of interest. The according measuring plan is depicted in Figure 3 where the time between epochs was about 15 minutes.

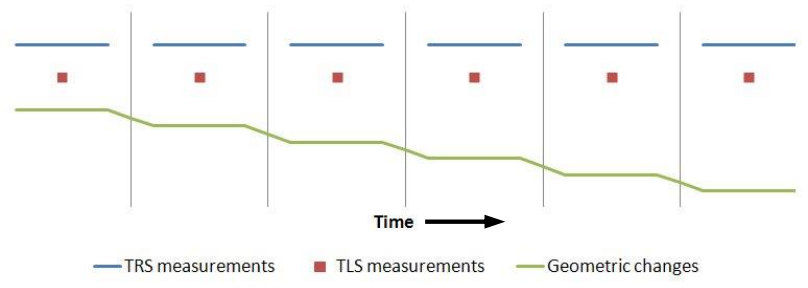

Figure 3: Measurement plan of the experiment -

TRS measurements (blue line), TLS measurements (red squares) and geometric changes (green line)

The slope has been scanned in every epoch with a point spacing of $20 \mathrm{~mm}$ at $45 \mathrm{~m}$ which means that areas that are further away are subject of coarser sampling and vice versa. Figure 4 illustrates the outcome of a deformation analysis [m] between the first and the last epoch derived from TLS measurements.

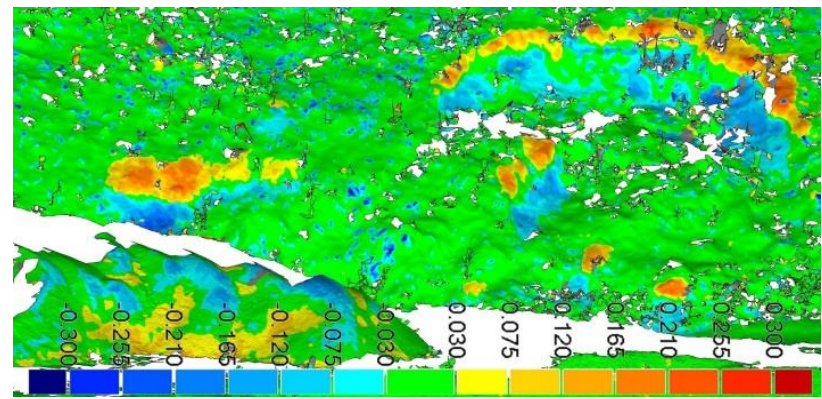

Figure 4: Deformation analysis [m] based on TLS measurements between reference and last epoch

\subsection{Data integration}

In order to achieve comparability between the two applied instruments' outcomes several steps need to be fulfilled. This is provoked by the fact that TRS only captures range changes in line of sight between epochs which are then converted into a 2D- displacement map while TLS capture Cartesian 3D point clouds. At first a transformation into a common coordinate frame needs to be conducted which usually contains scale, translation and rotation between the two systems. Since both sensors have been set plumb measured angles in the instruments' coordinate systems can be referred to as horizontal directions and vertical angles while rotations around the horizontal coordinate axes are omitted. As both sensors acquire metric data a scaling factor of 1 is assumed so that only four transformation parameters, $t_{x}, t_{y}, t_{z}$ and $r_{z}$, need to be determined. As a first step all Cartesian 3D-point clouds, namely $t_{0}$ to $t_{5}$, need to be translated into the coordinate system of the TRS. For this purpose differential GNSS measurements have been carried out on the standpoints of both instruments. The results are gathered in translation vector

$$
\boldsymbol{t}=\left[\begin{array}{lll}
t_{x} & t_{y} & t_{z}
\end{array}\right]^{T}
$$

An efficient way to apply the translation onto a $3 \mathrm{D}$ point

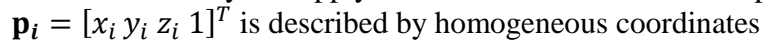

$$
\mathbf{T} \cdot \mathbf{p}_{\boldsymbol{i}}=\left[\begin{array}{cccc}
1 & 0 & 0 & t_{x} \\
0 & 1 & 0 & t_{y} \\
0 & 0 & 1 & t_{z} \\
0 & 0 & 0 & 1
\end{array}\right] \cdot\left[\begin{array}{c}
x_{i} \\
y_{i} \\
z_{i} \\
1
\end{array}\right]=\left[\begin{array}{c}
x_{i}+t_{x} \\
y_{i}+t_{y} \\
z_{i}+t_{z} \\
1
\end{array}\right]
$$

where translation matrix $\mathbf{T}$ contains the previously introduced translation vector $\mathbf{t}$. Subsequently the point clouds captured by TLS need to be adapted so that they are in geometric accordance to a 2D TRS displacement map. Initially the points of reference epoch $t_{0}$ are triangulated by applying GFaI's Final Surface software (GFAI 2013) in order to receive a closed surface representation. The remaining epochs are then converted into spherical coordinates which are assembled of the radius $r_{p}$

$$
r_{p}=\sqrt{x_{i}^{2}+y_{i}^{2}+z_{i}^{2}}
$$

the zenith angle $\vartheta$, which is described by the $z$-axis and the computation point vector $\mathbf{r}_{P}$

$$
\vartheta=\arctan \frac{\sqrt{x_{i}^{2}+y_{i}^{2}}}{z_{i}}
$$

as well as the azimuthal direction $\varphi$ between $x$-axis and the projection of $\mathbf{r}_{P}$ onto the $x$ - $y$-plane that can be calculated by

$$
\varphi=\arctan \frac{y_{i}}{x_{i}}
$$

Based on spherical coordinates distances between points $P$ from every epoch $t_{j}$ and the reference epoch, described by the surface model, can be computed along the computation point vector $\mathbf{r}_{P}=\left[\begin{array}{lll}r_{P} & \vartheta_{P} & \varphi_{P}\end{array}\right]^{T}$. Since the according $\vartheta$ and $\varphi$ are constant for the computation of the distance, only $\Delta r$ between reference surface and the according point needs to be determined. Therefore correspondences between surface segments and points need to be established. This is achieved by determining vectors between the origin of the TLS to each $3 \mathrm{D}$ point that 
intersect specific surface elements. In other words: if a vector intersects a triangle of the surface, then the according point of an epoch is corresponding to it. Based on three vertices $E_{i}$, that describe a triangle of the reference epoch, their according computation point vectors $\mathbf{r}_{i}=\left[r_{i} \vartheta_{i} \varphi_{i}\right]^{T}$ and a corresponding vector $\mathbf{r}_{P}$ the desired distance

$$
\Delta r_{p}=r_{p}-\left(\sum_{i=1}^{3} \frac{r_{i}}{\sqrt{\left(\vartheta_{p}-\vartheta_{i}\right)^{2}+\left(\varphi_{p}-\varphi_{i}\right)^{2}}}\right) /\left(\sum_{i=1}^{3} \frac{1}{\sqrt{\left(\vartheta_{p}-\vartheta_{i}\right)^{2}+\left(\varphi_{p}-\varphi_{i}\right)^{2}}}\right)
$$

can be computed. Since the origin of the TLS coincides with the origin of the TRS $\Delta r$ can be interpreted as geometric changes of the surface between reference and subsequent epochs. Hence these values should conform to the results derived from TRS. However, this assumption is only appropriate if the raster cell of the TRS is sufficiently small and if the vertical angle $\vartheta$ is determined in addition to the azimuth direction. While the current azimuth is known by an integrated stepping motor within the TRS this circumstance does not apply for the vertical angle. Thus all computed $\Delta r$ distances must be reduced to a two dimensional coordinate system. This is achieved by a projection of the point cloud onto a horizontal plane by omitting $\vartheta$ from $\mathbf{r}_{P}$

$$
\mathbf{r}_{P}=\left[r_{p} \vartheta \varphi\right]^{T} \rightarrow \mathbf{r}_{P}^{\prime}=\left[\begin{array}{ll}
r_{p} & \varphi
\end{array}\right]^{T}
$$

Finally all reduced $\Delta r$ values need to be assigned to according raster cells of the TRS, which equates to a horizontal rotation of the TLS data around the origin of the coordinate system. In order to achieve this corner cube reflectors have been applied which are distinctively visible within the RADAR imagery. Figure 5 illustrates the applied corner-cube reflectors.

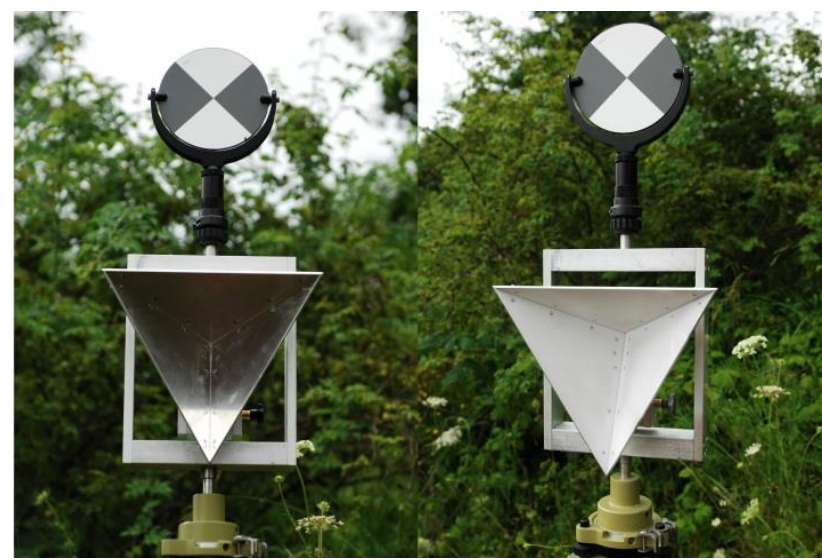

Figure 5: Target without (left) and with dulling spray (right)

The applied corner cube reflectors are assembled of three polished aluminium sheets with a longest side of $14 \mathrm{~cm}$ which leads to problems during data acquisition by laser scanning. Hence dulling spray has been applied to cut down the highly reflective characteristics that effected the reflectorless distance measurements by multi path effects. The centres of all targets have been determined by approximation of planes through each one of the metal sheets which have finally been intersected.
After correlation to a raster cell the average deformation of all points within each cell is computed. This yields to a point that lies within the centroid of each raster cell. Figure 6 illustrates the point cloud as acquired by TLS where the colours of each point indicate to which raster cell of the TRS they are associated with.

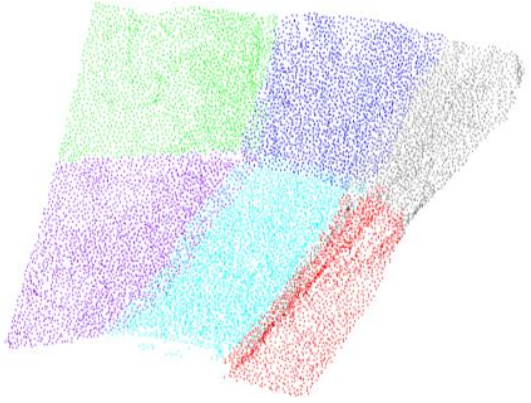

Figure 6: Mapping of TLS points to raster cells of the TRS

\subsection{Comparison of the outcome}

As a result of the procedure described in section 2.3 detected deformations by both instruments are now comparable. It can be notably seen that the results from TRS and TLS are in accordance which is illustrated in Figure 7 on example of a pixel where no geometric changes have been purposely conducted over all epochs.

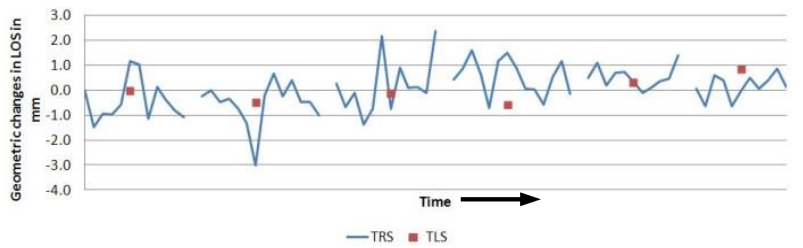

Figure 7: Comparison of TRS (blue) and TLS (red) measurements of a pixel where no geometric change has been conducted over all epochs

None of the captured epochs contained noteworthy deformations. Interestingly the variation of deformation that has been determined by TLS between epoch $t_{1}$ to $t_{5}$ amounts to less than $1 \mathrm{~mm}$. The reason for this circumstance can be found in averaging all points within the cell. Astonishingly the variation of continuously captured TRS data was about $+/-3 \mathrm{~mm}$ and hence much higher as the deviation detected by TLS. The reason for this behaviour can be found in the occasional traffic between slope and instruments within the pit. In spite of this impact the deviation amounts to less than $0.1 \mathrm{~mm}$ on average and median while the standard deviation of a single measurement sums up to $0.9 \mathrm{~mm}$. On the contrary the area within the pixel that is depicted in Figure 8 has been subject to deformation.

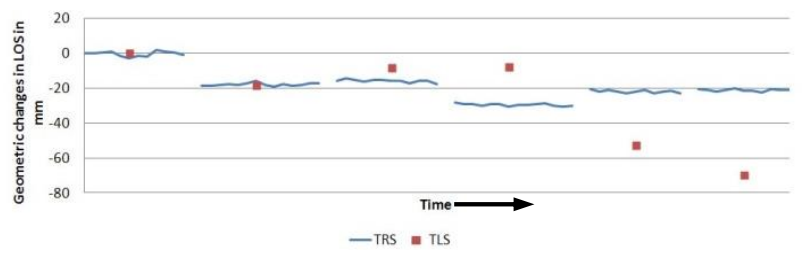

Figure 8: Comparison of TRS (blue) and TLS (red) derived deformations over all epochs in an area where large changes have been conducted 
Note that the scale of the vertical axis in this illustration is different to the one in Figure 7. A comparison of the time series which have been captured by TRS and TLS reveals large discrepancies from epoch $t_{2}$ onwards. This effect has been caused, as expected, by phase unwrapping during processing of TRS data.

\section{ASSISTED GROUND-BASED RADAR}

In order to eliminate the errors that were caused by ambiguities respectively phase unwrapping the outcome of TLS has been integrated into processing of TRS data which is referred to as assisted ground-based RADAR (aGB-RADAR). For the realisation of this procedure several computational steps must be conducted where the processed TLS data from 2.3 describes the point of origin. These datasets include range changes that occurred in a specific epoch $t_{i}$ in relation to the reference epoch and comply, in terms of resolution and geometry, with the TRS. At first these range changes need to be converted into phases $\phi_{L}$ of the TRS by applying the wavelength $\lambda$ of the used instrument

$$
\phi_{L}=2 \cdot \frac{2 \pi}{\lambda} \cdot \Delta r_{p}
$$

Subsequently $\phi_{L}$ is reduced to the residual $\Delta \phi_{L}$ by subtracting full periods so that the range of values lies between $-\pi$ and $+\pi$. Based on $\Delta \phi_{L}$, which has been derived from TLS, updated phases $\Delta \phi_{\text {update }}$ can be computed that eliminate phase unwrapping errors caused by the original residual $\Delta \phi_{\text {original }}$

$$
\Delta \phi_{\text {update }}=\Delta \phi_{\text {original }}-\Delta \phi_{L}
$$

Afterwards the updated residuals have been applied on the according values of the TRS for every epoch. Consequently the RADAR measurements have been reprocessed based on the updated residuals by using the commercial GAMMA software. Figure 9 depicts the reprocessed TRS measurements for the same area of the slope as illustrated in Figure 8. It can notably be seen that the values after spatial phase unwrapping are only ranging from $-3 \mathrm{~mm}$ to $+2 \mathrm{~mm}$ which leads to the conclusion that no more ambiguities occurred and that TLS and TRS are now in compliance. The median for the time series exemplified in Figure 9 amounts to $0.2 \mathrm{~mm}$, the average to $0.3 \mathrm{~mm}$ while the standard deviation sums up to $0.9 \mathrm{~mm}$.

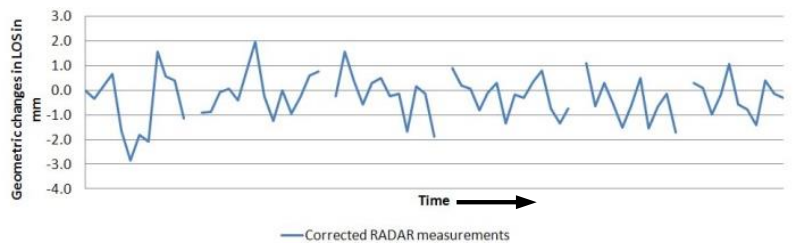

Figure 9: Corrected RADAR measurements of an area with deformations

As this precision correlates to a pixel that featured a geometrically stable area, as depicted in Figure 7 respectively discussed under section 2.4 , the correction process can be rated as successful.

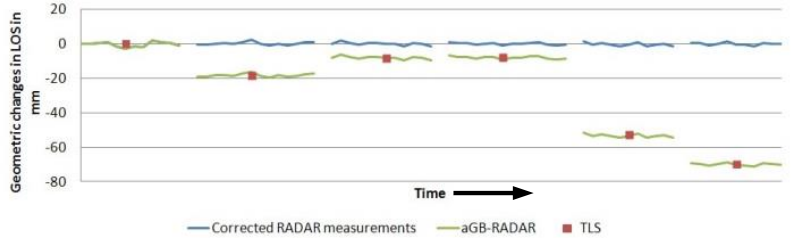

Figure 10: Outcome of aGB-RADAR for an area where large geometric changes occurred

Figure 10 depicts the final results: corrected TRS data is represented by blue lines, TLS measurements by red squares and the combined outcome derived by aGB-RADAR are denoted by green lines.

In order to avoid appliance of updated phases in areas where no significant geometric changes occurred TLS based deformation analysis is conducted. Therefore stochastic properties need to be assigned to the computed centroid of each raster cell for every epoch. A statistical test can then be carried out to clarify if the centroid of a raster cell has been subject of deformation or not. Since meaningful stochastic information of the applied instrument beyond the published range of $50 \mathrm{~m}$ is needed an experiment has been accomplished. A planar object has been scanned in $10 \mathrm{~m}$ increments ranging from $10 \mathrm{~m}$ to $110 \mathrm{~m}$. The experiment has been carried out indoors while the relative alignment between scanner and board as well as the scan resolution remained constant. Based on these observations stochastic behaviour of the reflectorless distance measurement unit has been derived. A polygonal function describes the dependence between variance of the distance measurement $\sigma_{\text {distance }}$ and the distance $d$ from applied TLS to the object which follows

$$
\sigma_{\text {distance }}=f \cdot d^{3}-g \cdot d^{2}+h \cdot d+i
$$

where $f$ amounts to $9.97301 \mathrm{E}-10, g$ to $-1.40861 \mathrm{E}-07, h$ to 8.64367E-06 and $i$ to $1.49108 \mathrm{E}-03$. A detailed description of the approach will follow in a separate publication. The Cartesian 3D coordinates of all TLS points are then converted into polar elements in order to being able to fulfil variancecovariance propagation (VCP). Therefore distance uncertainties have been computed as presented above while Leica specifies the angular precision of the applied TLS to 12" (LEICA 2013). After VCP was conducted single point accuracies are known for all 3D points, hence a point cloud where all points have stochastic information has been derived. Based on this information the unknown centroid $\hat{x}$, that is described by $\left[\begin{array}{lll}x_{c} & y_{c} & z_{c}\end{array}\right]^{T}$, of each cell including its stochastic information can be computed within a least squares adjustment. This procedure is in accordance with the computation of a weighted average. Therefore the covariance matrix $\Sigma_{l l}$ needs to be assembled as

$$
\Sigma_{l l}=\left[\begin{array}{ccc}
\sigma_{x_{1}}^{2} & \cdots & 0 \\
\vdots & \ddots & \vdots \\
0 & \cdots & \sigma_{z_{n}}^{2}
\end{array}\right]
$$

and contains variances of the coordinate components for each point. Correlations between 3D points are assumed to be nonexistent. The weight matrix $P$ can be computed by dividing $\Sigma_{l l}$ with the squared standard deviation of the unit weight and determining the inverse of the result. Cartesian 3D coordinates 
$x_{i}, y_{i}, z_{i}$, with $i=1, \ldots, n$, serve as observations which are stored within the observation vector 1

$$
\mathbf{l}=\left[\begin{array}{lllllll}
x_{1} & y_{1} & z_{1} & \cdots & x_{n} & y_{n} & z_{n}
\end{array}\right]^{T}
$$

while design matrix $\mathbf{A}$ for the weighted average follows

$$
\begin{gathered}
x \\
x_{1} \\
y_{1} \\
z_{1} \\
\vdots
\end{gathered}\left[\begin{array}{ccl}
1 & 0 & 0 \\
0 & 1 & 0 \\
0 & 0 & 1 \\
\vdots & \vdots & \vdots
\end{array}\right]
$$

The procedure is exemplified on the raster cell which is illustrated in Figure 8 for the reference and the last captured epoch. The precision derived by VCP of the according centre pixel amounts to $0.21 \mathrm{~mm}$ in $x, y$ and $z$ direction based on 475 points for the reference epoch. The according numbers for the last captured epoch are $0.26 \mathrm{~mm}$ derived from 507 points. Again variance-covariance propagation has been undertaken to transfer the stochastic information of the centroids from both epochs onto the spatial distance between them which leads to a standard deviation of $0.34 \mathrm{~mm}$. A t-test with a significance level of $5 \%$ is then conducted in order to clarify if a significant deformation occurred. This is obviously the case since a distance of $24.57 \mathrm{~cm}$ has been detected. Assuming similar stochastic properties and amount of points within a raster cell deformations larger than $0.39 \mathrm{~mm}$ are detectable by TLS which hence leads to computation of updated phases.

\section{CONCLUSION AND OUTLOOK}

A comparative study in a quarry has been carried out by applying a terrestrial laser scanner and a terrestrial radar scanner for the sake of deformation analysis. Geometric changes have been purposely introduced in local areas of a slope which were acquired by both instruments in several epochs. Processing of TLS and TRS data has at first been undertaken separately. In order to being able to compare the outcome of both systems, which represent several contrary properties e.g. being an absolute respectively relative measuring method, geometric conversion of the point cloud from TLS had to be conducted. The subsequent comparison revealed, as expected, that the results from both sensors do not comply with each other in regions where larger mass redistribution occurred. In summary it can be established that TRS measurements have been successfully combined with TLS data. Realistic results have been presented in regions of the area of investigation where conventional phase unwrapping approaches failed. Hence supportive usage of additional measurement systems provides the possibility to temporarily interrupt radar observations which would usually lead to problems during phase unwrapping. The procedure proposed in this contribution shows the potential of fusing TLS and TRS measurements which is referred to as assisted GB-RADAR (aGB-RADAR). By applying aGBRADAR the possibility arises to apply RADAR techniques for deformation monitoring conducted in temporarily interrupted campaigns. Prospective research will focus on accuracy assessment of the procedure and expansion to larger distances between instruments and object of interest by using mid- to long range TLS.

\section{REFERENCES}

Abellan, A., Jaboyedoff, M., Oppikofer, T., and Vilaplana, J. M., 2009. Detection of millimetric deformation using a terrestrial laser scanner: experiment and application to a rockfall event. Nat. Hazards Earth Syst. Sci., 9, 365-372.

Alba, M., Bernardini, G., Giussani, A., Rici, P., Roncoroni, F., Scaioni, M., Valgoi, P., Zhang, K., 2009. Measurement of dam deformations by terrestrial interferometric techniques. International Archives of Photogrammetry Remote Sensing: pp. 133-139.

Eling, D., 2009. Terrestrisches Laserscanning für die Bauwerksüberwachung. German Geodetic Commision, Volume C, No. 541, Munich, Germany.

GFaI, 2013. Final Surface ${ }^{\circledR}$ product page. www.final-surface.de (accessed 23.05.2013).

Hebel, H.-P., Knospe, S., Busch, W., 2011. Terrestrischer Radar-Scanner (TRS) - Ein neuartiges Instrument für die Böschungsüberwachung. In: Proceedings of GeoMonitoring 2011, Clausthal-Zellerfeld, Germany.

Kaasalainen, S., Hyyppä, J., Karjalainen, M., Krooks, A., Lyytikäinen-Saarenmaa, P., Holopainen, M., Jaakkola, A., 2010. Comparison of terrestrial laser scanner and synthetic aperture radar data in the study of forest defoliation. International archives of the ISPRS, pp. 82-87.

Leica, 2013. Leica ScanStation C10 datasheet. http://hds.leicageosystems.com/downloads 123/hds/hds/ScanStation\%20C10/br ochures-datasheet/Leica_ScanStation_C10_DS_us.pdf. (accessed 30.05.2013).

Lindenbergh, R., Pfeifer, N., 2005. A statistical deformation analysis of two epochs of terrestrial laser data of a lock, Proceedings of the 7th Conference on Optical 3-D Measurement Techniques, Vienna, Austria, pp. 61-70.

Luzi, G., 2010. Ground based SAR interferometry: A novel tool for geoscience. Imperatore, P. Riccio, D. (Eds.), Geoscience and Remote Sensing. New Achievements, Intech (2010), pp. 1-26.

Rödelsperger, S., 2011. Real-time Processing of Ground Based Synthetic Aperture Radar (GB-SAR) Measurements. German Geodetic Commision, Volume C, No. 668, Munich, Germany.

Werner, C., Strozzi, T., Wiesmann, A., Wegmüller, U., 2008. A real-aperture radar for ground-based differential interferometry . Proceedings of the 2008 IEEE International Geoscience and Remote Sensing Symposium, Boston, USA.

Wiesmann, A., Werner, C., Strozzi, T., Wegmüller, U., 2008. Measuring deformation and topography with a portable radar interferometer. Proceedings of the $13^{\text {th }}$ FIG Symposium on deformation measurement and analysis, Lisbon, Portugal.

Wujanz, D., Krueger, D., Neitzel, F., 2013. DefoScan++: Surface based registration of terrestrial laser scans for deformation monitoring. Proceedings of the JISDM conference, Nottingham, Great Britain.

\section{ACKNOWLEDGEMENTS}

The first author has been supported by the German Federal Ministry of Education and Research (BMBF) listed under the support code 17N0509. 